\title{
The LATIN multiscale computational method and the Proper Generalized Decomposition
}

\author{
P. Ladevèze ${ }^{\mathrm{a}, \mathrm{b}}$, J.-C. Passieux ${ }^{\mathrm{a}}$, D. Néron ${ }^{\mathrm{a}}$ \\ ${ }^{a}$ LMT-Cachan (ENS Cachan/CNRS/UPMC/PRES UniverSud Paris) \\ 61, avenue du Président Wilson, F-94235 CACHAN CEDEX, France \\ \{ladeveze, passieux, neron\}@lmt.ens-cachan.fr \\ ${ }^{b}$ EADS Foundation Chair 'Advanced Computational Structural Mechanics '
}

\begin{abstract}
This paper deals with the synergy between the LATIN multiscale method and what is called the Proper Generalized Decomposition (PGD) which is the key of its performances.

Key words: time and space multiscale, PGD, LATIN, domain decomposition, parallel computing, homogenization
\end{abstract}

\section{Introduction}

Today, in structural mechanics, there is a growing interest in a class of techniques called "multiscale computational approaches", which are capable of analyzing structures in which two or more very different scales can be identified [8, 9, 10,3]. A typical engineering example is that of a relatively large structure in which local cracking or local buckling occurs. Another typical engineering problem is related to the increasing interest in material models described on a scale smaller than that of the macroscopic structural level, with applications ranging from the design of composite materials and structures to manufacturing [15]. In such situations, the structure being studied is highly heterogeneous and the local solution involves short-wavelength phenomena in both space and time. As a result, classical finite element codes lead to systems with very large numbers of degrees Preprint submitted to Computer Methods in Applied of freedom and the corresponding calculation costs are generally prohibitive. Therefore, one of today's main challenges is to derive computational strategies capable of solving such engineering problems through true interaction between the two scales in both space and time: the microscale and the macroscale.

A new micro/macro computational strategy was proposed in [18] which involved space and time homogenization while avoiding the drawbacks of classical homogenization theory [23]. Here, it will be described in detail for viscoelastic materials and unilateral contact with or without friction, a case already introduced in [16]. More complex types of material behavior could also be taken into account [17].

The first point of the method consists in splitting the space domain into an assembly of substructures and interfaces ; and the time interval into coarse sub-intervals. Mechanics and Engineering 
The separation between the macroscale and the microscale takes place at the interfaces only. Each quantity of interest is considered to be the sum of a macro quantity and a micro quantity, where the macro quantities are defined as "mean values" in time and in space.

The second characteristic of the method is the use of the LATIN method, a nonincremental iterative computational strategy applied over the entire time interval [14]. At each iteration, one must solve a macro problem defined over the entire structure and coarse sub-intervals, along with a family of independent linear micro problems, substructure wise.

The paper is focused on the third and main characteristic of the method which concerns the resolution, over the time-space domains, of micro problems within the substructures. The fact that they are global over the time-space domain could be seen as a drawback of the LATIN method. On the contrary: this is a great advantage ; it allows through a variational formulation over the time-space domain to handle radial time-space approximation which enters in what is called the Proper Generalized Decomposition or, in short, PGD. Such approximation reduces calculation and storage cost drastically and presents some similarities with the POD. Initially introduced for the analyze and reduction of statistical and experimental data, the a posteriori decomposition techniques, also known as Karhunen-Loeve Expansion, Singular Value decomposition or Principal Component Analysis, are now used in the context of model reduction [11, 22, 4]. But those methods require a partial, total or even approximate resolution of the reference problem in a preliminary stage called learning phase or snapshot. Then a truncated POD of the snapshot is used to reduce the initial model. In opposition with this first class of methods, the PGD, initially called "radial time-space approximation" [12] is an a priori resolution technique which is driving to a POD decomposition without requiring any basis nor known solutions. The solution is built thanks to the resolution of a few spatial problems (time independent) and temporal problems (scalar ODE). This technique has already been used in the context of stochastic problems [19] and multidimensional problems $[1,2,6]$.

In this paper a new, more efficient and more robust version is proposed for the resolution of the micro problems of the multiscale strategy, in the case of material model with internal variables. Several numerical examples, among which a rather large scale heterogeneous structure with multicracks, will illustrate the capabilities of the proposed approach.

\section{Description of the problem}

In this Section, a brief review of the main aspects of the multiscale computational strategy is presented. Further details can be found in $[14,17]$.

\subsection{Reference problem}

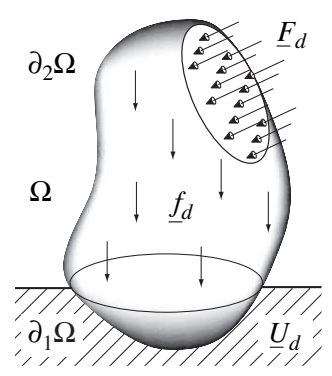

Figure 1: Reference problem on $\Omega$ 
For the sake of simplicity, let us consider the quasi-static and isothermal evolution of a viscoelastic structure defined over the time-space domain $[0, T] \times \Omega$, under the assumption of small perturbations (for a much larger range of material behaviors see $[13,17])$. This structure is subjected to prescribed body forces $\underline{f}_{d}$, to traction forces $\underline{F}_{d}$ over a part $\partial_{2} \Omega$ of the boundary, and to prescribed displacements $\underline{U}_{d}$ over the complementary part $\partial_{1} \Omega$ (see Figure 1 ).

Let $\varepsilon$ denote the strain associated with displacement field $\underline{U}$ and $\boldsymbol{\sigma}$ stress. The strain is devided into an elastic part $\varepsilon_{\mathrm{e}}$ verifying the state law $\boldsymbol{\sigma}=\mathbf{K} \boldsymbol{\varepsilon}_{\mathrm{e}}$ (K Hooke operator), and an inelastic part $\varepsilon_{\mathrm{p}}$ which linked to the stress through the state evolution law $\dot{\varepsilon}_{\mathrm{p}}=\mathbf{B}(\boldsymbol{\sigma})$ which is possibly non-linear. The displacement, strains and stresses are subjected to initial conditions at $t=0$.

\subsection{Sub-structuration of the problem}

The structure is viewed as an assembly of simple components, i.e. substructures and interfaces [14] (see Figure 2). Concerning time, the domain $[0, T]$ is split into a few coarse sub-intervals $I_{i}^{C}=\left[t_{i}^{C}, t_{i+1}^{C}\right]$. The in-

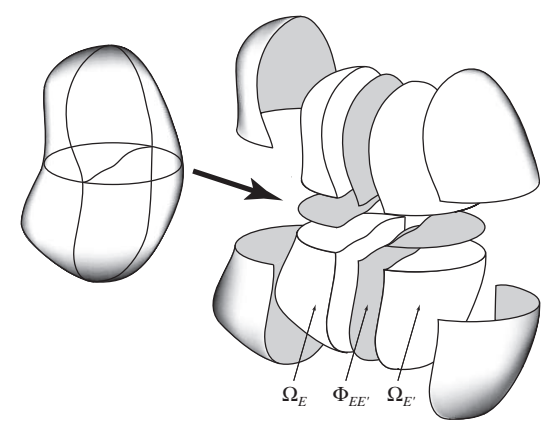

Figure 2: Dcomposition of $\Omega$ into substructures $\Omega_{E}$ and interfaces $\Phi_{E E^{\prime}}$

terface between one substructure $\Omega_{E}{ }^{1}$ and

\footnotetext{
${ }^{1}$ the notation $\square_{E}$ is used to indicate the restriction of a quantity $\square$ to the substructure $\Omega_{E}$
}

one substructure $\Omega_{E^{\prime}}$ is denoted by $\Phi_{E E^{\prime}}$ (Fig. 2). Each substructure and each interface have their own variables and equations (admissibility, balance or constitutive relation) driving their evolution. The state of one substructure $\Omega_{E}$ is entirely defined by $\dot{\varepsilon}_{\mathrm{p} E}$ and $\boldsymbol{\sigma}_{E}$, the restriction of the fields $\dot{\varepsilon}_{\mathrm{p}}$ and $\boldsymbol{\sigma}$ on $\Omega_{E}$. The state of an interface $\Phi_{E E^{\prime}}$ is given by $\underline{W}_{E}$ the restriction of displacement $\underline{U}_{E}$ on $\Phi_{E E^{\prime}} ;$ and $\underline{F}_{E}$, the normal stress on $\Phi_{E E^{\prime}}$.

Let $\mathbf{s}_{E}=\left(\dot{\varepsilon}_{\mathrm{p} E}, \dot{W}_{E}, \boldsymbol{\sigma}_{E}, \underline{F}_{E}\right)$ denote the set of fields describing the state of the substructure $\Omega_{E}$ and its boundary $\partial \Omega_{E}$ and $\mathbf{E}_{E}, \mathcal{W}_{E}, \mathbf{F}_{E}$ and $\mathcal{F}_{E}$ denote the corresponding spaces. For sake of simplicity, we only present the case of a null initial condition except from the initial displacement $\underline{U}_{E \mid t=0}$, but taking into account other kinds of initial condition is not difficult.

\subsection{Admissibility conditions for substruc- ture $\Omega_{E}$}

Let us introduce the following spaces, as well as their corresponding vector spaces (denoted by $\square^{\star}$ ):

- the space $\mathcal{E}_{E}$ of kinematic admissible fields $\left(\dot{\varepsilon}_{E}, \underline{W}_{E}\right)$ :

$$
\begin{gathered}
\left(\dot{\varepsilon}_{E}, \underline{W}_{E}\right) \in \mathcal{E}_{E} \Longleftrightarrow \\
\exists \underline{U}_{E}, \quad \underline{U}_{E \mid \partial \Omega_{E}}=\underline{W}_{E} \\
\underline{U}_{E \mid t=0}=\underline{U}_{E 0}, \quad \varepsilon_{E}=\nabla_{s y m} \underline{U}_{E}
\end{gathered}
$$

- the space $\mathcal{F}_{E}$ static admissible fields 
$\left(\boldsymbol{\sigma}_{E}, \underline{F}_{E}\right):$

$$
\begin{aligned}
& \left(\boldsymbol{\sigma}_{E}, \underline{F}_{E}\right) \in \mathcal{F}_{E} \Longleftrightarrow \forall\left(\dot{\varepsilon}_{E}^{\star}, \underline{W}_{E}^{\star}\right) \in \mathcal{E}_{E}^{\star}, \\
& \quad-\int_{I_{i}^{C} \times \Omega_{E}} \boldsymbol{\sigma}_{E}: \dot{\varepsilon}_{E}^{\star} d \Omega d t \\
& \quad+\int_{I_{i}^{C} \times \Omega_{E}} \underline{f}_{d} \cdot \underline{\dot{U}}_{E}^{\star} d \Omega d t \\
& \quad+\int_{I_{i}^{C} \times \partial \Omega_{E}} \underline{F}_{E} \cdot \underline{\dot{W}}_{E}^{\star} d S d t=0
\end{aligned}
$$

- the space $\mathbf{A}_{\mathbf{d} E}$ of "E-admissible" variables $\mathbf{s}_{E}$ :

$$
\begin{aligned}
& \mathbf{s}_{E}=\left(\dot{\varepsilon}_{\mathrm{p} E}, \dot{W}_{E}, \boldsymbol{\sigma}_{E}, \underline{F}_{E}\right) \in \mathbf{A}_{\mathbf{d} E} \Longleftrightarrow \\
& \left(\mathbf{K}^{-1} \dot{\boldsymbol{\sigma}}_{E}+\dot{\varepsilon}_{\mathrm{p} E}, \underline{W}_{E}\right) \in \mathcal{E}_{E} \quad \text { and } \\
& \left(\boldsymbol{\sigma}_{E}, \underline{F}_{E}\right) \in \mathcal{F}_{E}
\end{aligned}
$$

The interface is characterized by the restriction to $\Phi_{E E^{\prime}}$ of the displacement fields $\left(\underline{W}_{E}, \underline{W}_{E^{\prime}}\right)$, and of the force fields $\left(\underline{F}_{E}, \underline{F}_{E^{\prime}}\right)$. Those restrictions are denoted by $\left(\underline{W}_{E E^{\prime}}, \underline{W}_{E^{\prime} E}\right)$ and $\left(\underline{F}_{E E^{\prime}}, \underline{F}_{E^{\prime} E}\right)$ :

A relation between those quantities is introduced to characterize the behavior $\mathbf{b}_{E E^{\prime}}$ of the interface.

$$
\mathbf{b}_{E E^{\prime}}\left(\underline{\dot{W}}_{E E^{\prime}}, \underline{\dot{W}}_{E^{\prime} E}, \underline{F}_{E E^{\prime}}, \underline{F}_{E^{\prime} E}\right)=0
$$

For example a perfect interface: $\underline{\underline{W}}_{E E^{\prime}}=$ $\underline{\dot{W}}_{E^{\prime} E}$ et $\underline{F}_{E E^{\prime}}+\underline{F}_{E^{\prime} E}=\underline{0}$.

The philosophy of the method is clearly to make the interfaces coincide with the complex surface behaviors of the problem like cohesive interfaces or frictional contact. The boundary conditions are also viewed as particular interface behaviors.

\subsection{Two scale description of the unknowns}

This multiscale approach consists in introducing a two-scale description of the unknowns. These two scales are denoted "macro" $\left(\square^{M}\right)$ and "micro" $\left(\square^{m}\right)$. This choice is physically sound: macro part are mean values in space and time. For $\underline{\underline{W}}_{E} \in$ $\mathcal{W}_{E}$, its macro part $\underline{\dot{W}}_{E}^{M}$ and micro complement $\underline{W}_{E}^{m}$ are defined by $\forall \underline{F}^{M \star} \in \mathcal{F}_{E}^{M}$ :

$$
\begin{array}{r}
\int_{I_{i}^{C} \times \partial \Omega_{E}}\left(\dot{W}_{E}^{M}-\underline{\dot{W}}_{E}\right) \cdot \underline{F}^{M \star} d S d t=0 \\
\text { and } \quad \underline{\dot{W}}_{E}^{m}=\underline{\dot{W}}_{E}-\underline{\dot{W}}_{E}^{M}
\end{array}
$$

The spaces $\mathcal{F}_{E}^{M}$ and $\mathcal{W}_{E}^{M}$ can be chosen arbitrarily. In practice, they are defined by the linear part of force and displacement in space Fig.3, and quadratic part in time FIG.4.

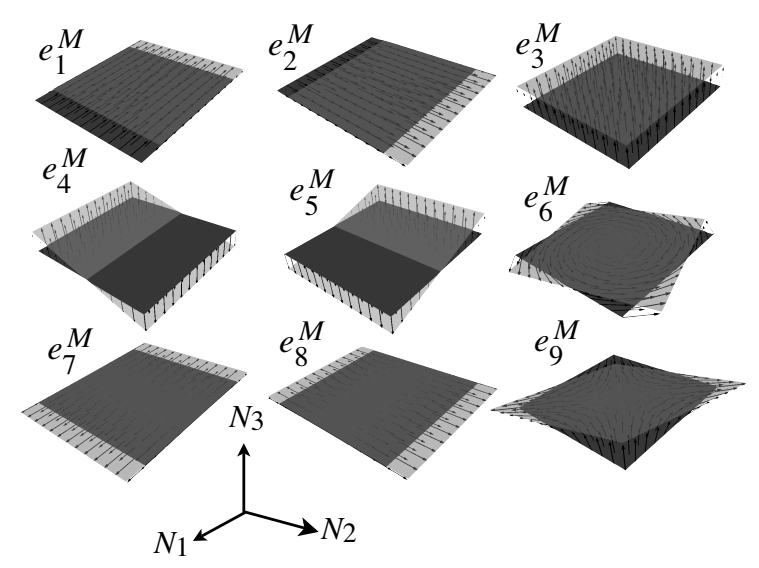

Figure 3: Linear macro space basis for a square interface $\Phi_{E E^{\prime}}$ in the $3 \mathrm{D}$ case

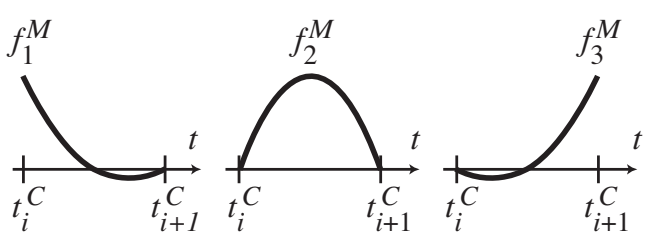

Figure 4: Quadratic macro time basis on $I_{i}^{C}$

An important point of the strategy, which provides its multiscale character, is the choice of the admissibility conditions for the macro quantities. The set of the macro forces $\underline{F}^{M}=\left(\underline{F}_{E}^{M}\right)_{\Omega_{E} \subset \Omega}$ is required, a priori, to verify the transmission condi- 
tions systematically, including the boundary conditions. The corresponding subspace of $\mathcal{F}^{M}=\bigotimes \mathcal{F}_{E}^{M}$ is designated by $\mathcal{F}_{a d}^{M}$. The subspace of $\mathcal{F}$ whose elements have their macro parts in $\mathcal{F}_{\text {ad }}^{M}$ is designated by $\mathcal{F}_{\text {ad }}$. We use the definition for $\mathcal{W}^{M}, \mathcal{W}_{a d}^{M}$ and $\mathcal{W}_{a d}$.

\section{The multiscale computational strategy}

\subsection{The LATIN method}

The engine of the strategy is the LATIN method [14]. It is a general iterative non linear solver for time-dependant problems, which works globally over the entire timespace domain. It is said to be nonincremental since it allows to deal with a problem which is defined with a variational form over the entire time-space domain which makes possible to use different resolution techniques, such as the PGD which is nonincremental.

The first principle of the LATIN method is to separate the solutions of the equations into two subspaces: The space $\mathbf{A}_{\mathbf{d}}$ of solutions to the global linear equations: $\mathbf{s}=\left(\mathbf{s}_{E}\right)_{\Omega_{E} \subset \Omega} \in \mathbf{A}_{\mathbf{d}}$ if $\mathbf{s}$ verifies

a) the $E$-admissibility condition: $\forall \Omega_{E} \subset \Omega, \quad \mathbf{s}_{E} \in \mathbf{A}_{\mathbf{d} E}$

b) the admissibility of macro forces: $\underline{F} \in \mathcal{F}_{\text {ad }}$

And the space $\boldsymbol{\Gamma}$ of solutions to the local non-linear equations: $\hat{\mathbf{s}}=\left(\mathbf{s}_{E}\right)_{\Omega_{E} \subset \Omega} \in \boldsymbol{\Gamma}$ if $\hat{\mathbf{s}}$ verifies

c) the evolution law on $\Omega_{E}$ : $\forall \Omega_{E} \subset \Omega, \quad \dot{\varepsilon}_{\mathrm{p} E}=\mathbf{B}\left(\boldsymbol{\sigma}_{E}\right)$

c) the interface behavior $\forall \Phi_{E E^{\prime}}$ : $\mathbf{b}_{E E^{\prime}}\left(\underline{\dot{W}}_{E E^{\prime}}, \underline{\dot{W}}_{E^{\prime} E}, \underline{F}_{E E^{\prime}}, \underline{F}_{E^{\prime} E}\right)=0$

The state law and initial conditions are verified by the solutions of both $\mathbf{A}_{\mathbf{d}}$ and $\boldsymbol{\Gamma}$.
Clearly, the exact solution to the decomposed problem is defined by:

$$
\mathbf{s}_{\text {exact }} \in \mathbf{A}_{\mathbf{d}} \cap \mathbf{\Gamma}
$$

The second principle of the method is to use a two-stage iterative scheme to obtain the solution to the problem. Indeed, one it-

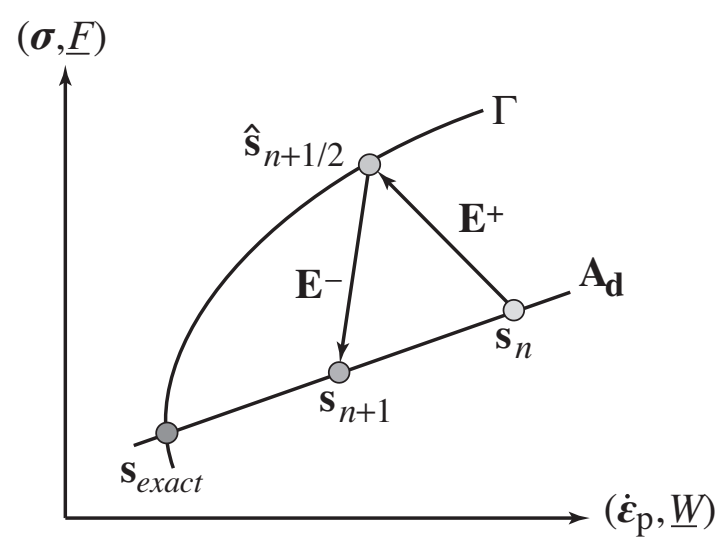

Figure 5: One iteration of the LATIN methode

eration of this solver is made of two stages called "local stage" and "linear stage". As shown on figure 5 these stages consists in building a member of $\boldsymbol{\Gamma}$ and $\mathbf{A}_{\mathbf{d}}$ alternatively. On figure 5 one can notice, that, in order to close the problem, we need to introduce what we call the "search directions" $\mathbf{E}^{+}$and $\mathbf{E}^{-}$which will be detailed later.

The third principle of the method is to take advantage of the time-space framework by using adapted approximations of the unknowns. More precisely, we use the PGD to solve the problems of the linear stage. This technique will be presented in details in the section 4.

\subsection{Local stage}

Local stage consists in building $\hat{\mathbf{s}}_{n+1 / 2} \in$ $\Gamma$ knowing $\mathbf{s}_{n} \in \mathbf{A}_{\mathbf{d}}$ and using a search direction $\mathbf{E}^{+}$followed by $\left(\hat{\mathbf{s}}_{E, n+1 / 2}-\mathbf{s}_{E, n}\right)$ and 
defined by (subscript $E$ skipped):

$$
\mathbf{E}^{+}\left\{\begin{array}{l}
\left(\dot{\hat{\varepsilon}}_{p}-\dot{\varepsilon}_{\mathrm{p}}\right)+\mathbf{H}(\hat{\boldsymbol{\sigma}}-\boldsymbol{\sigma})=0 \\
(\underline{\hat{\hat{W}}}-\underline{\dot{W}})-\mathbf{h}(\underline{\hat{F}}-\underline{F})=0
\end{array}\right.
$$

where $\mathbf{H}$ and $\mathbf{h}$ are symmetric definite operators which are the parameters of the method. At this stage, the problems are possibly non linear but local-i.e. on each discretization point - in space (should $\mathbf{B}$ be also local) and often in time, and lend themselves to the highest degree of parallelism.

\subsection{Linear stage}

This stage consists in building $\mathbf{s}_{n+1} \in \mathbf{A}_{\mathbf{d}}$ knowing $\hat{\mathbf{s}}_{n+1 / 2} \in \boldsymbol{\Gamma}$ and using the search direction $\mathbf{E}^{-}$, followed by $\left(\mathbf{s}_{E, n+1}-\hat{\mathbf{s}}_{E, n+1 / 2}\right)$ (see Figure 5). This stage is solved incrementally over the coarse sub-intervals. The substructure part of the search direction is defined by:

$$
\left(\dot{\varepsilon}_{\mathrm{p}}-\dot{\hat{\varepsilon}}_{p}\right)-\mathbf{H}(\boldsymbol{\sigma}-\hat{\boldsymbol{\sigma}})=0
$$

Because of the admissibility of the macro forces, the boundary part of the search direction is written using a weak formulation, introducing a Lagrange multiplier $\underline{\widetilde{W}}_{E}^{M}$ to guarantee the admissibility of the macro forces in a weak sense: $\underline{\widetilde{W}}^{M}=\left(\dot{\widetilde{W}}_{E}^{M}\right)_{\Omega_{E} \subset \Omega} \in$ $\mathcal{W}_{\mathrm{ad}}^{M \star}$ :

$$
\begin{aligned}
& \forall \underline{F}^{\star} \in \mathcal{F}, \sum_{\Omega_{E} \subset \Omega}\left\{\int _ { I _ { i } ^ { C } \times \partial \Omega _ { E } } \left(\left(\underline{\dot{W}}_{E}-\underline{\hat{\hat{W}}}_{E}\right)+\right.\right. \\
& \left.\left.\mathbf{h}\left(\underline{F}_{E}-\underline{\hat{F}}_{E}\right)-\underline{\hat{W}}_{E}^{M}\right) \cdot \underline{F}_{E}^{\star} d S d t\right\}=0
\end{aligned}
$$

and the admissibility of the macro forces is expressed by:

$$
\begin{aligned}
& \quad \dot{\vec{W}}^{M \star} \in \mathcal{W}_{\mathrm{ad}}^{M \star}, \\
& \sum_{\Omega_{E} \subset \Omega}\left\{\int_{I_{i}^{C} \times \partial \Omega_{E}} \underline{\dot{\vec{W}}}_{E}^{M \star} \cdot \underline{F}_{E} d S d t\right. \\
& \left.\int_{I_{i}^{C} \times \partial \Omega_{E} \cap \partial_{2} \Omega}-\dot{\overrightarrow{\hat{W}}}_{E}^{M \star} \cdot \underline{F}_{d} d S d t\right\}=0
\end{aligned}
$$

At the linear stage, $\mathbf{s}_{E}$ verifies the $E$ admissibility and the search direction $\mathbf{E}^{-}$. The admissibility of kinematic variables can be written in a weak form by:

$$
\begin{aligned}
& \forall\left(\boldsymbol{\sigma}_{E}^{\star}, \underline{F}_{E}^{\star}\right) \in \mathcal{F}_{E}^{\star}, \\
& \int_{I_{i}^{C} \times \Omega_{E}} \boldsymbol{\sigma}_{E}^{\star}:\left(\mathbf{K}^{-1} \dot{\boldsymbol{\sigma}}_{E}+\dot{\boldsymbol{\varepsilon}}_{\mathrm{p} E}\right) d \Omega d t \\
& \quad+\int_{I_{i}^{C} \times \partial \Omega_{E}} \underline{F}_{E}^{\star} \cdot \underline{\underline{W}}_{E} d S d t=0
\end{aligned}
$$

\subsubsection{The micro problem}

The micro problem is given by introducing the search direction $\mathbf{E}^{-}$(EQ.(7)(8)) in the previous weak formulation (10) and defined by:

$$
\begin{aligned}
& \text { Find }\left(\boldsymbol{\sigma}_{E}, \underline{F}_{E}\right) \in \mathcal{F}_{E}, \text { such that, } \\
& \forall\left(\boldsymbol{\sigma}_{E}^{\star}, \underline{F}_{E}^{\star}\right) \in \mathcal{F}_{E}^{\star}, \\
& \int_{I_{i}^{C} \times \Omega_{E}} \boldsymbol{\sigma}_{E}^{\star}:\left(\mathbf{K}^{-1} \dot{\boldsymbol{\sigma}}_{E}+\mathbf{H} \boldsymbol{\sigma}_{E}\right) d \Omega d t \\
& +\int_{I_{i}^{C} \times \partial \Omega_{E}} \underline{F}_{E}^{\star} \cdot \mathbf{h} \underline{F}_{E} d S d t= \\
& \int_{I_{i}^{C} \times \Omega_{E}} \boldsymbol{\sigma}_{E}^{\star}:\left(\mathbf{H} \hat{\boldsymbol{\sigma}}_{E}-\dot{\hat{\varepsilon}}_{\mathrm{p}}\right) d \Omega d t \\
& +\int_{I_{i}^{C} \times \partial \Omega_{E}} \underline{F}_{E}^{\star} \cdot\left(\underline{\hat{\hat{W}}}_{E}+\mathbf{h} \underline{\hat{F}}_{E}+\underline{\hat{W}}_{E}^{M}\right) d S d t
\end{aligned}
$$

The unknowns $\varepsilon_{\mathrm{p} E}$ and $\underline{W}_{E}$ can be deduced from the former thanks to the search di- 
turned into a primal problem trough a duality transformation. It depends on the quantities of the previous local stage $\hat{\mathbf{s}}_{E}$, on the known quantities $f_{d}$, on the initial conditions and on $\underline{\tilde{W}}_{E}$ which is unknown at this stage. Thanks to the linearity of the problem, its solution can be viewed as the following sum:

$$
\mathbf{s}_{E}=\mathbf{s}_{E}\left(\dot{\widetilde{W}}_{E}^{M}\right)+\hat{\hat{\mathbf{s}}}_{E}
$$

Where $\hat{\hat{\mathbf{s}}}_{E}$ is the solution to the problem (11) when $\underline{W}_{E}=0$. This solution can be computed, because it depends on fields that are known at this stage. The solution $\tilde{\mathbf{s}}_{E}$ linearly depends on $\widetilde{W}_{E}^{M}$ which is unknown. In particular, the previous relations also applies to the macro forces:

$$
\underline{F}_{E}^{M}=\mathbf{L}_{E}{\dot{\vec{W}}_{E}^{M}}^{M}+\underline{\hat{\hat{F}}}_{E}^{M}
$$

Where $\underline{\hat{F}}_{E}^{M}$ is the macro part of the effort of $\hat{\mathbf{s}}_{E}$, and $\mathbf{L}_{E}$ is a linear operator which can be interpreted as a homogenized operator over time-space subdomain $\Omega_{E} \times I_{i}^{C}$. It can be computed and its computational cost is reasonable since the macro quantities belong to a small-sized finite dimension space. Its computation requires the resolution of a set of micro problems with null initial conditions, no additional forces, with $\hat{\mathbf{s}}_{E}$ equal to zero, and $\dot{\widetilde{W}}_{E}^{M}$ taking the value of the basis vectors of $\mathcal{W}_{E}^{M}$ alternatively. This operator only depends on the choice of the macro basis and on the parameters of the search directions $\mathbf{h}$ and $\mathbf{H}$. Therefore, it can be constant over many iterations if $\mathbf{h}$ and $\mathbf{H}$ are not changed.

\subsubsection{The macro problem}

The macro problem, build by the introduction of the homogenized operator (13) into the EQ.(9), is defined by: find ${\underline{\dot{\widehat{W}}^{M}}}^{M} \in$ $\mathcal{W}_{\text {ad }}^{M}$ such that, $\dot{\vec{W}}^{M \star} \in \mathcal{W}_{\text {ad }}^{M \star}$,

$$
\begin{aligned}
& \sum_{\Omega_{E} \subset \Omega}\left\{\int_{I_{i}^{C} \times \partial \Omega_{E}}{\underline{\tilde{W}_{E}}}_{E}^{\star} \cdot \mathbf{L}_{E} \dot{\widetilde{W}}_{E}^{M}-\right. \\
&\left.\dot{\widetilde{W}}_{E}^{M \star} \cdot\left(\underline{F}_{d}-\underline{\hat{\hat{F}}}_{E}^{M}\right) d S d t\right\}=0
\end{aligned}
$$

This linear time-space problem is define over the whole interfaces and entire coarse sub-interval $I_{i}^{C}$. One can prove that this problem has a unique solution if the boundary conditions are taken into account trough an interface behavior. The macro problem yields $\underline{\widetilde{W}}^{M}$ and, trough a micro resolution, $\tilde{\mathbf{s}}$. Then one can determine s completely.

\subsection{Convergence of the algorithm}

If the constitutive relation operator $\mathbf{B}$ is monotonous and if the interface are perfect or unilateral contact without friction, the multiscale strategy verifies the usual assumptions of the convergence proof of the LATIN method (see [14]). Since the solution $\mathbf{s}_{\text {ref }}$ is the intersection of $\mathbf{A}_{\mathbf{d}}$ and $\boldsymbol{\Gamma}$, a measure of the distance between $\hat{\mathbf{s}}_{n+1 / 2}$ and $\mathbf{s}_{n+1}$ is a good error indicator to verify the convergence of the strategy. For example, we can use:

$$
\eta^{2}=\frac{\left\|\hat{\mathbf{s}}_{n+1 / 2}-\mathbf{s}_{n+1}\right\|^{2}}{\left\|\hat{\mathbf{s}}_{n+1 / 2}+\mathbf{s}_{n+1}\right\|^{2}}
$$

with:

$$
\begin{aligned}
& \|\mathbf{s}\|^{2}=\frac{1}{2} \sum_{\Omega_{E}} \sum_{I_{i}^{C}} \\
& \int_{\Omega_{E} \times I_{i}^{C}}\left(\boldsymbol{\sigma}_{E}: \mathbf{K}^{-1} \boldsymbol{\sigma}_{E}+\dot{\varepsilon}_{\mathrm{p} E}: \mathbf{K} \dot{\boldsymbol{\varepsilon}}_{\mathrm{p} E}\right) d \Omega d t+ \\
& \int_{\partial \Omega_{E} \times I_{i}^{C}}\left(\underline{F}_{E} \cdot \mathbf{h} \underline{F}_{E}+\underline{\underline{W}}_{E} \cdot \mathbf{h}^{-1} \underline{\underline{W}}_{E}\right) d S d t
\end{aligned}
$$


In order to ensure the convergence of the algorithm for a larger range of behaviors, we classically modify the linear stage by a relaxation stage. We rename $\breve{\mathbf{s}}_{n+1}$ the quantity previously denoted by $\mathbf{s}_{n+1}$ and we define $\mathbf{s}_{n+1}$ with the relation:

$$
\mathbf{s}_{n+1}=\mu \breve{\mathbf{s}}_{n+1}+(1-\mu) \mathbf{s}_{n}
$$

where $\mu$ is a relaxation parameter usually taken equal to 0.8 .

\section{The Proper Generalized Decompo- sition}

Along iterations, one has to solve a set of linear micro problems (11) defined on the time-space substructures, which can vary only slightly from an iteration to the other. Classical incremental integration techniques do not take advantage of this property and the cost of this stage can be prohibitive. This observation triggered the development of what is called the PGD.

This approximation, introduced by [12] and initially called "radial approximation" (see also [14]), is part of the LATIN method. It has been shown in previous works [20], that it can lead to drastic savings in terms of computational costs. The basic idea is to approximate a function $f$ defined over a space-time domain $\Omega \times I_{i}^{C}$ by the sum of the products of separated variables functions: a time function $\lambda_{i}(t)$ and a space function $\Lambda_{i}(\underline{M}): \forall(t, \underline{M}) \in I_{i}^{C} \times \Omega$,

$$
f(t, \underline{M}) \approx f_{m}(t, \underline{M})=\sum_{i=1}^{m} \lambda_{i}(t) \Lambda_{i}(\underline{M})
$$

where the products $\lambda_{i}(t) \Lambda_{i}(\underline{M})$ are called "radial time-space functions". It is important to note that this is not a classical spectral decomposition because neither the $\lambda_{i}$ nor the $\Lambda_{i}$ are known a priori.
Such a decomposition makes this method present some similarities with the Proper Orthogonal Decomposition (POD [5]). However, classical POD-based methods (see for example [21, 4, 22]) are different from the PGD since they need to know a priori one of the two families of functions $\left(\lambda_{i}\right.$ or $\Lambda_{i}$ ). To build this family of functions, they usually require a total, partial or even approximate resolution of the reference problem (called snapshot) with standard incremental techniques. Then a POD of the snapshot is computed, the corresponding basis is truncated and fixed (for example $\Lambda_{i}$ ), and finally the reduced problem consists in finding the other family of functions (in this case $\lambda_{i}$ ). Furthermore, with classical POD methods, the minimum quantity of information needed (namely the snapshot) and the minimum order of reduction required (namely the truncation) to build the most relevant but reduced model, can not be known a priori. On the contrary, the PGD can be viewed as a truly a priori resolution technique, since both $\lambda_{i}$ and $\Lambda_{i}$ are unknown and computed at the same time. One $m^{\text {th }}$ order approximation can be enriched easily at any time.

The PGD has been successfully applied to more general problems with different denominations: "Generalized Spectral Decomposition" for stochastic problems in [19], by replacing the time variable by a stochastic one ; "separated representation technique" for multivariable problems in $[1,2,6]$, by adding new coordinates in the separated representation.

\subsection{Rewriting of a micro problem over $I_{i}^{C} \times$ $\Omega_{E}$ at iteration $n$}

In this part, we rewrite the linear stage as a problem of minimization on the correction of the solution, in which the ap- 
proximation will be introduced. First, let us remark that the search direction can be rewritten in terms of correction of the solution $\Delta \mathbf{s}_{n}=\mathbf{s}_{n}-\mathbf{s}_{n-1} \in \mathbf{A}_{\mathbf{d}}^{\star}$ :

$$
\begin{array}{r}
\Delta \dot{\varepsilon}_{p n}-\mathbf{H} \Delta \boldsymbol{\sigma}_{n}+\bar{\Delta}_{n}=0 \\
\Delta \underline{\dot{W}}_{n}+\mathbf{h} \Delta \underline{F}_{n}-\underline{\bar{\delta}}_{n}=0
\end{array}
$$

with the following given quantities :

$$
\begin{aligned}
\bar{\Delta}_{n}= & \mathbf{H}\left(\hat{\boldsymbol{\sigma}}_{n-1 / 2}-\boldsymbol{\sigma}_{n-1}\right)- \\
& \left(\dot{\hat{\varepsilon}}_{p, n-1 / 2}-\dot{\boldsymbol{\varepsilon}}_{p, n-1}\right) \\
\overline{\boldsymbol{\delta}}_{n}= & \left(\underline{\widehat{\hat{W}}}_{n-1 / 2}-\underline{\dot{W}}_{n-1}\right)+ \\
& \mathbf{h}\left(\underline{\widehat{F}}_{n-1 / 2}-\underline{F}_{n-1}\right)+\dot{\widehat{W}}^{M}
\end{aligned}
$$

For each $I_{i}^{C} \times \Omega_{E}$, the search direction (19) can be interpreted as a linear constitutive relation. Thus, an equivalent formulation consists in minimizing the global constitutive relation error in $\mathbf{A}_{\mathbf{d} E}^{\star}$, which is defined over the time-space substructure $I_{i}^{C} \times \Omega_{E}$. Then, the linear stage consists in finding $\Delta \mathbf{s}_{E, n}$ which minimizes on $\mathbf{A}_{\mathbf{d} E}^{\star}$ the constitutive relation error $e_{C R, E}^{2}\left(\mathbf{s}_{E, n}\right)$ associated with the search direction, defined by (subscript $n$ skipped):

$$
\begin{array}{r}
e_{C R, E}^{2}\left(\Delta \mathbf{s}_{E}\right)=\left\|\Delta \dot{\varepsilon}_{p E}-\mathbf{H} \Delta \boldsymbol{\sigma}_{E}+\bar{\Delta}\right\|_{\mathbf{H}, E}^{2} \\
+\left\|\Delta \underline{\dot{W}}_{E}+\mathbf{h} \Delta \underline{F}_{E}-\underline{\bar{\delta}}\right\|_{\mathbf{h}, E}^{2}(20)
\end{array}
$$

where the corresponding norms are:

$$
\|\square\|_{\mathbf{H}, E}^{2}=\int_{I_{i}^{C} \times \Omega_{E}}\left(1-\frac{t}{T}\right) \square \circ \mathbf{H}^{-1} \square d \Omega d t
$$

and:

$$
\|\square\|_{\mathbf{h}, E}^{2}=\int_{I_{i}^{C} \times \partial \Omega_{E}}\left(1-\frac{t}{T}\right) \square \cdot \mathbf{h}^{-1} \square d S d t
$$

Let us notice, that if we find an approximation of this problem, the only equation that will not be exactly verified is the search direction which is a parameter of the method, and which has an effect on the convergence rate only. According to our tests, even with a coarse verification of the search direction, the effect on the convergence rate is rather slight.

\subsection{Introduction of the approximation}

We classically choose to approximate with this technique the correction $\Delta \mathbf{s}$ only. The choice of the approximation presented herein is an improvement over the version introduced in [20]. The starting point is the introduction as unknowns of the radial time-space approximations of the corrections related to the inelastic strain and to the interface displacement:

$$
\begin{aligned}
& \Delta \varepsilon_{\mathrm{p}}(t, \underline{M})=\sum_{k=1}^{p} a^{k}(t) \mathbf{E}_{\mathbf{p}}^{k}(\underline{M}) \\
& \Delta \underline{W}(t, \underline{M})=\sum_{k=1}^{p} a^{k}(t) \underline{Z^{k}}(\underline{M})
\end{aligned}
$$

Using the $E$-admissibility conditions, one determines the other quantities of interest in terms of the previous unknowns:

$$
\begin{gathered}
\Delta \varepsilon(t, \underline{M})=\sum_{k=1}^{p} a^{k}(t) \mathbf{E}^{k}(\underline{M}) \\
(\Delta \boldsymbol{\sigma}, \Delta \underline{F})(t, \underline{M})=\sum_{k=1}^{p} a^{k}(t)\left(\mathbf{C}^{k}, \underline{G}\right)(\underline{M})
\end{gathered}
$$

where the space function $\mathbf{E}^{k}$ is computed thanks to the resolution of an annex problem, which consists in seeking $\underline{U}^{k} \in \mathcal{U}^{2}=$ $\left\{\underline{U} \in H^{1}\left(\Omega_{E}\right)\right.$, suchthat $\left.\left.\underline{U}\right|_{\Gamma}=\Delta \underline{Z}^{k}\right\}$, $\forall \underline{U}^{\star} \in H_{0}^{1}\left(\Omega_{E}\right)$

$$
\int_{\Omega_{E}} \varepsilon\left(\underline{U}^{\star}\right): \mathbf{K}\left(\varepsilon\left(\underline{U}^{k}\right)-\mathbf{E}_{p}^{k}\right) d \Omega=0
$$

Then the strain is deduced by $\mathbf{E}^{k}=\varepsilon\left(\underline{U}^{k}\right)$, and the stress given by: 
the interface forces $\underline{G}^{k}$ deduced by:

$$
\underline{G}^{k}=\left.\mathbf{C}^{k} \underline{n}\right|_{\partial \Omega_{E}}
$$

and the space operators are defined through standard finite element approximation over the space domain $\Omega_{E}$.

As we will see in the examples, compared to the previous version of the radial loading time-space approximation, we obtain the same quality of approximation with only half the number of time functions.

This technique can be easily extended to other material models with internal variables, which represent a rather large range of materials. In this case, the total number of unknown is 1 for the an-elastic strain rate and 1 for each addition internal variables.

\subsection{First order approximation}

In order to solve the problem of minimization on $e_{R C, E}^{2}\left(\Delta \mathbf{s}_{E}\right)(20)$, the idea is to seek minima alternatively with respect to time and to space, thanks to Algorithm 1:

- initialisation $a^{0}(t)$ (for instance $f(t)=\alpha t)$

for $k=1, \ldots, k_{\max }$ do

- Spatial problem: the time function $a^{k}(t)$ is known, one seeks $\left(\mathbf{E}_{p}^{k}, \underline{Z}^{k}\right)$ minimizing $e_{C R, E}^{2}\left(\Delta \mathbf{s}_{E}\right)$

- Normalization of space functions.

- Time problem: the space functions $\left(\mathbf{E}_{p}^{k}, \underline{Z}^{k}\right)$ is known, one seeks $a^{k}(t)$ minimizing $e_{C R, E}^{2}\left(\Delta \mathbf{s}_{E}\right)$

\section{end}

Algorithm 1: iterative resolution of the first order approximation

The minimization with respect to time variable leads to a scalar differential equation defined on $I_{i}^{C}$, whose resolution is quite cheap. The minimization with respect to time variable leads to the resolution of a few classical finite element problems defined on $\Omega_{E}$ and time-independants. According to our examples, Algorithm 1 converges very quickly, therefore, in practice, $k_{\max }$ is taken equal to 2 or 3 only.

\section{4. $m^{\text {th }}$ order approximation}

In order to computer a $m^{\text {th }}$ order approximation, the pairs are built one by one. Let us consider that a $m^{\text {th }}$ order approximation has already been computed. All the known pairs are chosen to be fixed, and the $(m+1)^{t h}$ pair is computed with Algorithm 1.

\subsection{Exemple}

In order to exemplify the performances of the approximation, we now consider the resolution of a micro problem defined on a single 3D L-shaped substructure $\Omega_{E}$ over $I_{i}^{C}=[0, T]$ (Only one coarse time subinterval). The structure is only subjected to

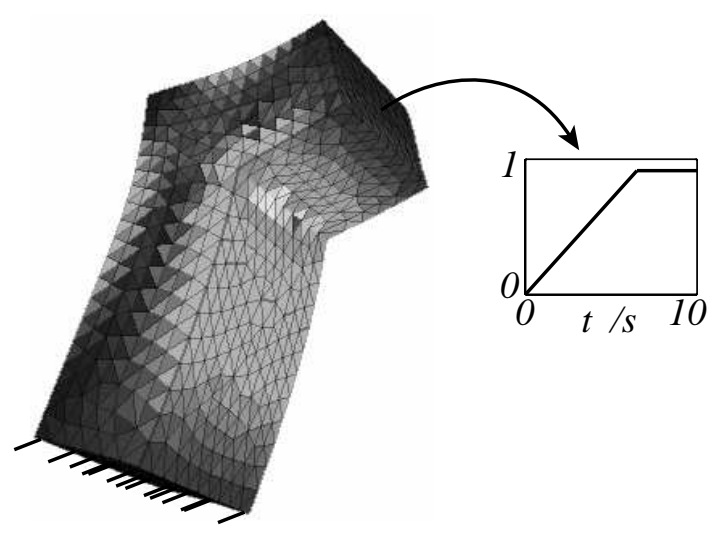

Figure 6: Definition the geometry and loading.

the distribution of $\underline{\widetilde{W}}$ defined on Figure 6. It is homogeneous and made of the viscoelastic material whose Young modulus E, poisson ratio $\nu$ and viscosity parameter $\eta$ are $E=50 \mathrm{GPa}, \nu=0.3$ and $\eta=10 \mathrm{~s}$. The res- 

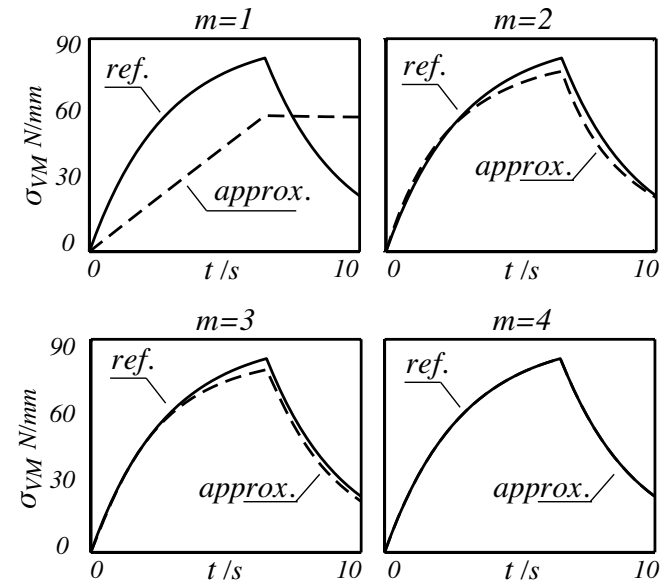

Figure 7: Quality of the approximation.

olution of this problem with the technique proposed herein is compared to the technique presented in [20] and to the classical incremental technique associated with a discontinuous Galerkin scheme in time. Figure 7 presents the evolution on $I_{i}^{C}$ of the maximum stress in one element of the structure for different orders of approximation (from order $m=1$ up to $m=4$ ). We can notice, that even with a low-order approximation, we have a rather good accuracy. With only a fourth order approximation, the difference can not be seen in the bare eye. In order to

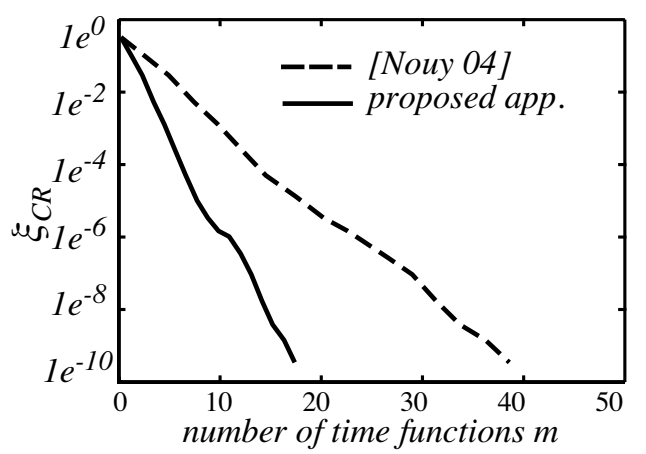

Figure 8: Convergence of the approximation.

quantify the convergence of the approximation, Figure 8 presents the evolution of the relative constitutive relation error defined by:

$$
\xi_{C R}^{2}(\mathbf{s})=\frac{\sum_{\Omega_{E}} e_{C R, E}^{2}\left(\Delta \mathbf{s}_{E}\right)}{\left\|\Delta \mathbf{s}_{E}\right\|^{2}}
$$

versus the number of time functions $m$ for the technique presented here and the previous one presented in [20]. We can see that the approximation converges very quickly. By the way, for this example, the new approximation technique presented in this paper need twice less time functions to reach a same level of error. The method proposed here seems more efficient and more robust than the previous one. Figure 9 displays the first four spaces radial functions corresponding to the resolution of this problem and their corresponding time functions. One can notice that the radial functions are adapted to the structure and its loading, this is reason why so few functions are necessary to get a very accurate approximation.

\subsection{Practical use of this technique in the strategy}

Since the construction of the space functions is by far the most expensive step of this process, it is advantageous to store and reuse these functions (see $[20,17]$ ). Thus, the space functions constructed up to Iteration $n-1$ are reused systematically during Iteration $n$. Let us note that a reduced basis can be shared by several substructures if these substructures are similar.

Let us assume that we are dealing with Iteration $n$ of the multiscale strategy and that we have at our disposal a reduced basis made up of the space functions $\left\{\left(\mathbf{E}_{\mathbf{p}}^{k}, \underline{Z}^{k}\right)\right\}_{k=1, \ldots, m}$ for the approximation of the corrections related to the inelastic strain $\Delta \varepsilon_{\mathrm{p} E}$ and interface displacement $\Delta \underline{W}_{E}$. The space functions related to the other quantities $\Delta \varepsilon_{E}, \Delta \sigma_{E}$ and $\Delta \underline{F}_{E}$ are also 

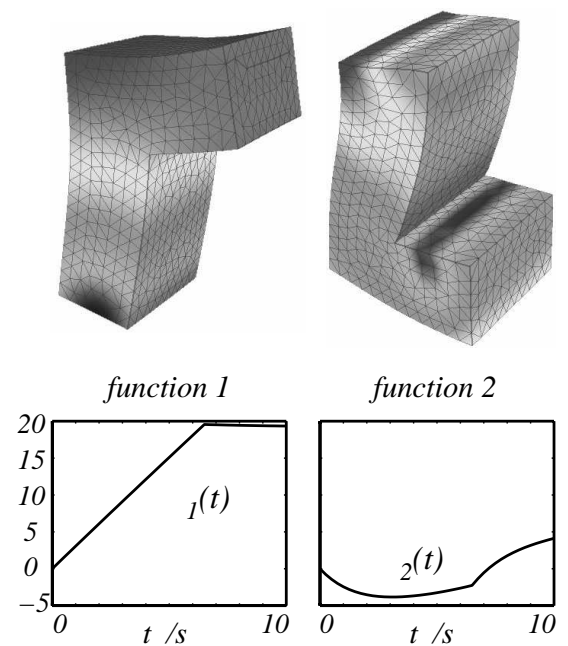

function 2
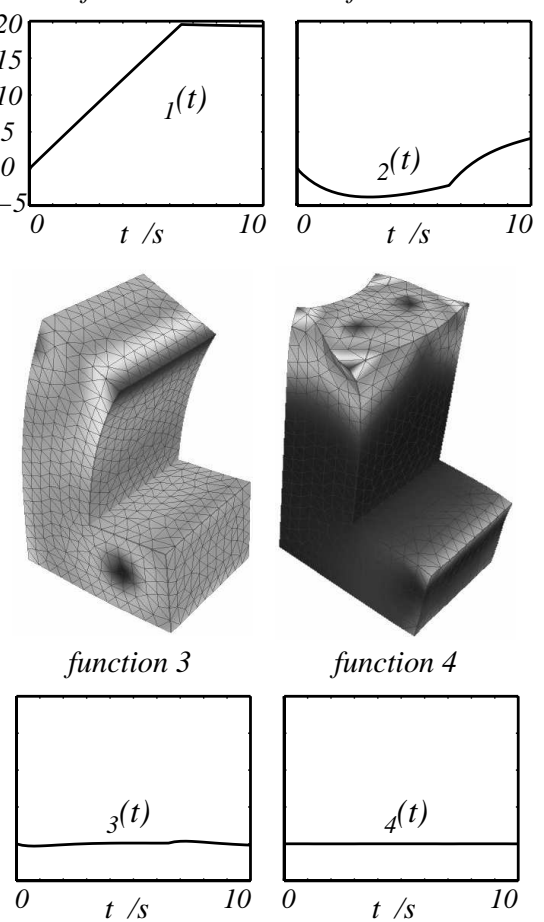

function 4

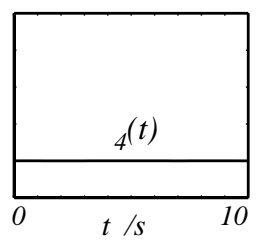

Figure 9: Four first radial functions.

considered to be known. One proceeds Iteration $n$ of the LATIN as follows :

Step 1: use of the reduced basis. This phase consists in building a cheap prediction thanks to the knowledge of the resolution at previous iterations. The approximation is introduced into the constitutive relation error (20), but here, only the time functions are the unknowns. In other words, one seeks the best combination of the reduced basis of space functions which minimizes the constitutive relation error $e_{C R, E}^{2}\left(\Delta \mathbf{s}_{E}\right)$. These time func- tions verify a system of linear differential equations in time with conditions at $t=0$ and $t=T$, whose solution is obtained classically. This is generally a rather small system. An error indicator base on the constitutive relation error $\xi_{C R, E}$ is now computed to quantify the accuracy of this first prediction. If its value is greater than a critical $\xi^{0}$, then the linear stage at Iteration $n+1$ is considered to be solved, and switch to the next iteration. Otherwise, one proceeds to Step 2.

Step 2: adding new functions. The prediction previously computed is considered to be known, and a new first order approximation is sought. Once this pair of time and space function is calculated, the space function is orthogonalized and added to the reduced basis of space functions.

At each iteration, for each substructure, a maximum of one function is computed.

\section{Example of the multiscale strategy with the radial approximation}

Let us consider the evolution over [0,10 s] of a 3D composite structure containing cracks (see Figure 10). The structure is made of a 4D Carbon/Carbon composite consisting of four reinforcement directions parallel to the largest diagonals of a cube. These materials are used in the throat nozzles of solid propulsion systems owing to their excellent thermo-mechanical properties [15]. The structure is clamped at the bottom and subjected to prescribe forces $\underline{F}$ (see Figure 10). The cracks are described using unilateral contact with Coulomb friction characterized by Parameter $f=0.3$.

The structure consists of two types of cells with different orientations of the fibers. The cells are heterogeneous and consist of a 


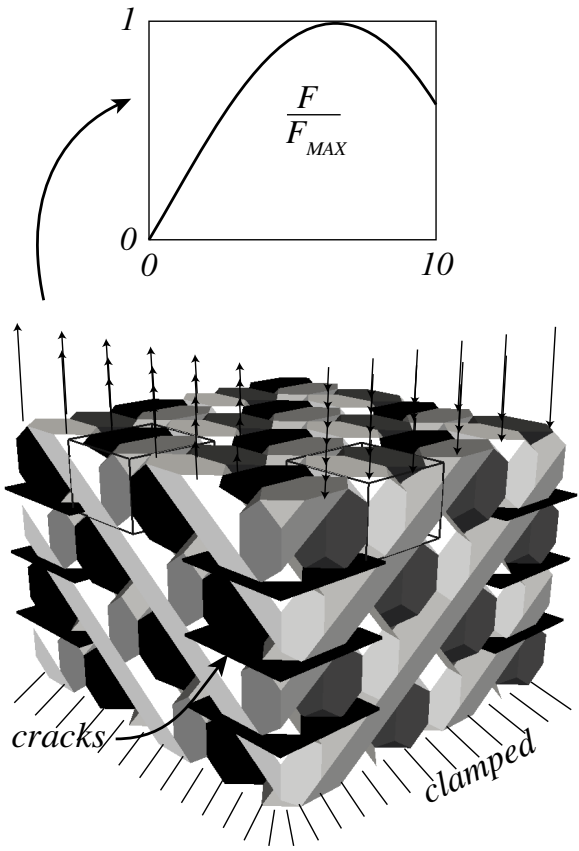

Figure 10: Decomposition and microscale discretizations in space

matrix with four fibers oriented in 4 directions. The matrix and the fibers are linear viscoelastic and their properties are given in Table 1. The corresponding constitutive relations are $\dot{\boldsymbol{\varepsilon}}_{p}=\mathbf{B}_{i} \boldsymbol{\sigma}=\frac{1}{\eta_{i}} \mathbf{K}_{i}^{-1} \boldsymbol{\sigma}$.

\begin{tabular}{lll}
\hline & Matrix & Fiber \\
\hline Young & $E_{1}=50 \mathrm{GPa}$ & $E_{2}=250 \mathrm{GPa}$ \\
Poisson & $\nu_{1}=0.3$ & $\nu_{2}=0.2$ \\
Viscosity & $\eta_{1}=10 \mathrm{~s}$ & $\eta_{2}=1000 \mathrm{~s}$ \\
\hline
\end{tabular}

Table 1: Material properties

The problem was divided into 64 substructures and 204 interfaces as shown on Figure 11, each substructure corresponding to one cell. On the microlevel, Type-I and Type-II substructures and Type-I and Type-II interfaces were meshed with 847, 717 and 144 DOFs respectively. The macro part consisted of a single linear element with only 9 DOFs per interface. With respect to time, the microlevel was associated with a refined discretization into 60 intervals using a zero-order discontinuous Galerkin scheme, and the macrolevel was associated with one single coarse sub-interval using a secondorder discontinuous Galerkin scheme.

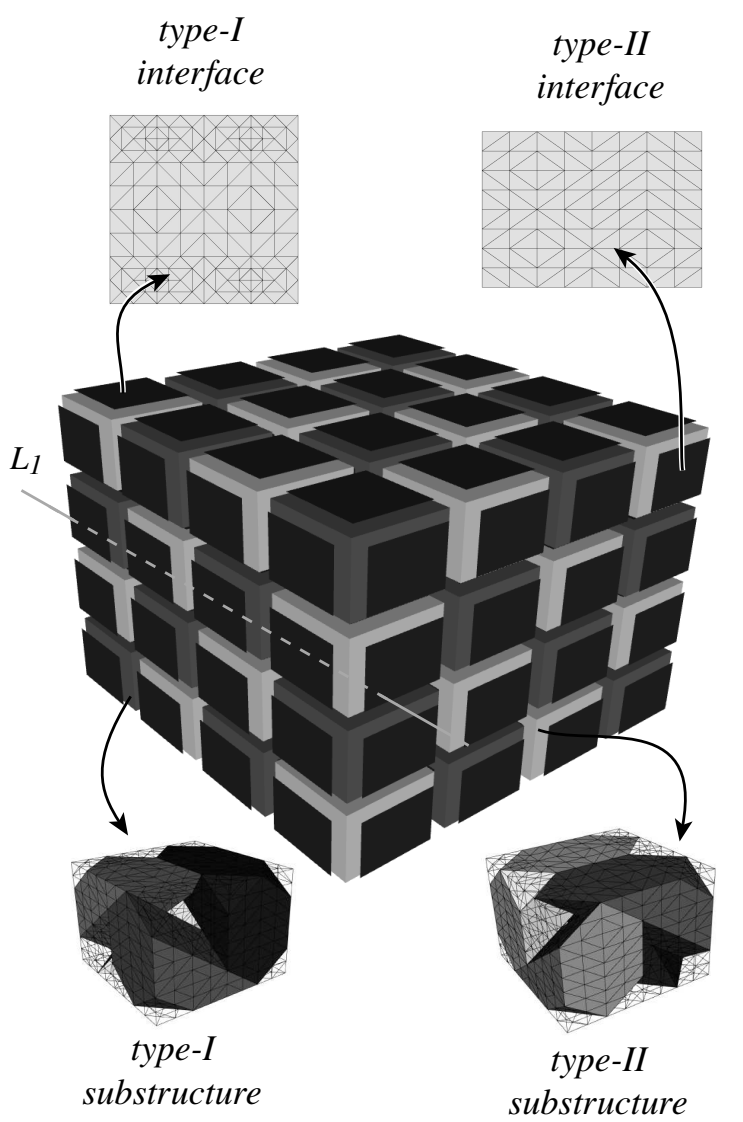

Figure 11: Definition of the problem and its loading

Since, here, the state evolution law is linear, the search direction chosen for the substructures was $\mathbf{H}=\mathbf{B}$. The characteristic length of the interfaces being $L_{E}=4 \mathrm{~mm}$, we chose for all the interfaces the search direction $\mathbf{h}=h \mathbf{I}$, where $h=\frac{L_{E}}{E_{1} \nu_{1}}$ is a constant scalar.

Figure 12 shows the evolution of the LATIN residual indicator $\eta$ throughout the iterations. One can observe that the algorithm converges rapidly toward 


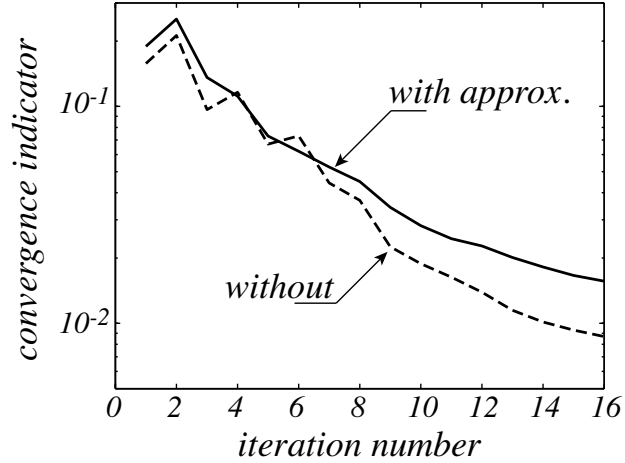

Figure 12: Convergence of the method

an accurate solution (1\% error after less than 20 iterations). On this figure is also plotted the convergence of the method without approximation. We can see that the approximation is quite robust, and make the multiscale strategy converge, despite the huge savings in terms of computational costs. There exists 8 reduced basis each one shared by 8 substructures of the same type. The evolution of the average size of the reduced basis over iterations is given Figure 13. The algorithm requires, in average by reduced basis, the computation of less than 5 functions per iterations to approximate the 2 micro problems of 60 time-steps of the 8 substructures.

In this example, the CPU time taken by the local stage is neglectable (less than $1 \%$ of the total time, since the linear stage involves 3D FE resolutions). However, the interfaces play also an important role through the macro problem for which the cost could be expensive. Indeed, when the number of subdomains increases, the size of the macro problem, which is global on the whole set of interfaces, increases too. In this case, alternative techniques have been developed to solve the macro problem, thanks to the introduction of a third scale [18].
Concerning the performances of the PGD approximation, it is better to compare the strategy with and without PGD in term of the total number of subdomains space resolutions. Thanks to the PGD technique, this number is approximately divided by 20 and the storage cost divided by 200 .

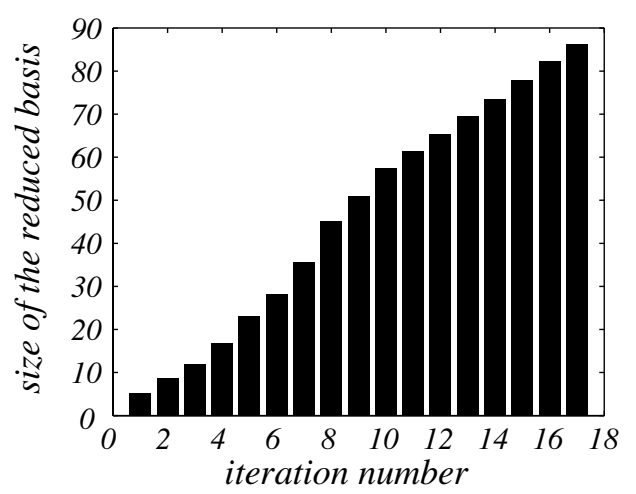

Figure 13: Size of the reduced basis over the iterations

Figure 14 shows the approximate solution over the structure at the final time $T=10 \mathrm{~s}$ for Iterations 1, 5, 10 and 15 and after convergence (the reference solution). One can observe that thanks to the resolution of a macro problem the method leads, even after a few iterations, to a rather good approximation of the solution to the problem over both the space and time domains. After a tenth of iterations, the solution becomes even more accurate and the stress and displacement discrepancies tend to zero. After 20 iterations, the difference between the approximate solution and the reference solution is no longer visible.

An example of the micro/macro description of the solution is given in Figures 15 and 16. Figure 15 show the evolutions of Force $\underline{F}$ and its macro part $\underline{F}^{M}$ respectively at time $t=2 / 3 T$ over a horizontal line $L_{1}$, and as functions of time at one point of the previous line. Figures 16 show the 

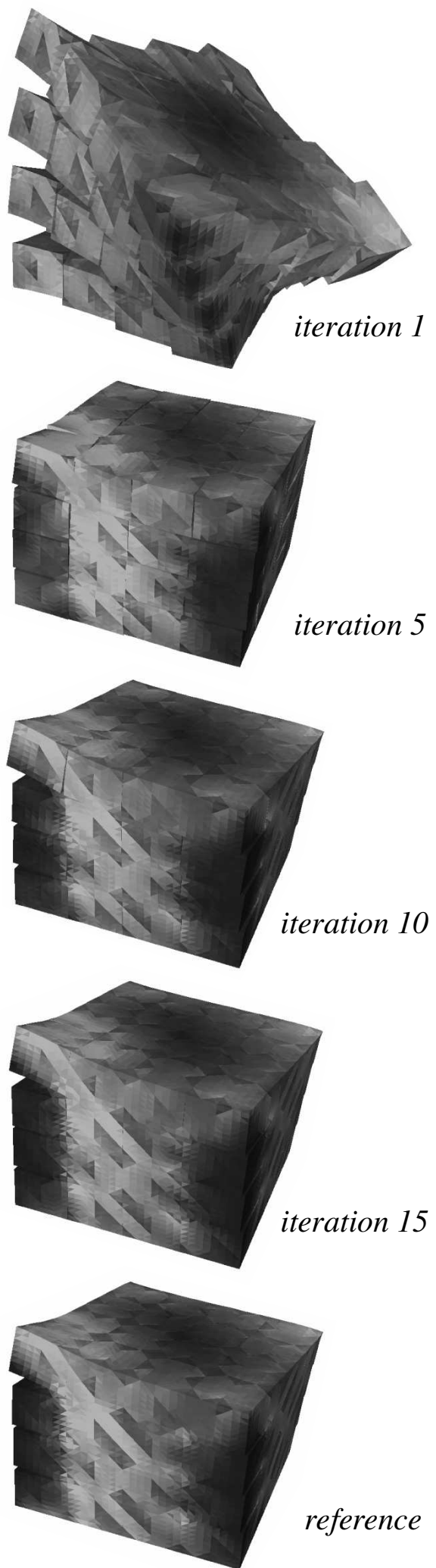

Figure 14: Approximate solutions throughout the iterations same evolutions for Displacement $\underline{W}$ and its macro part $\underline{W}^{M}$. One can observe
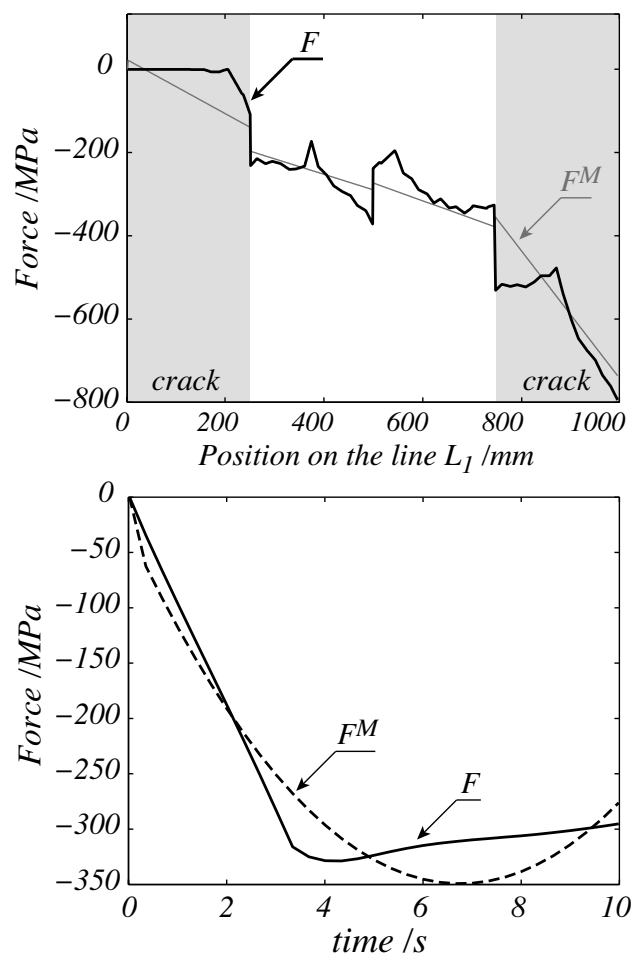

Figure 15: $\underline{F}$ and $\underline{F}^{M}$ over Line $L_{1}$ and over $[0, T]$

that the macro part of the quantities being considered constitutes a good average approximation of the solution, obtained with only a very small number of basis functions (27 DOFs per interface and per coarse subinterval). The choice of such a basis leads to the resolution at each iteration of a macro problem with a strong mechanical meaning and with only a few DOFs (in this example, 6,480 DOFs compared to approximately 300,000 DOFs for the assembled reference problem).

Here, the method is illustrated with a linear behavior in the subdomains with interface nonlinearities. It could be extended to other more complex constitutive relations, such as plasticity, visco-plasticity... such as described in $[13,7]$. 

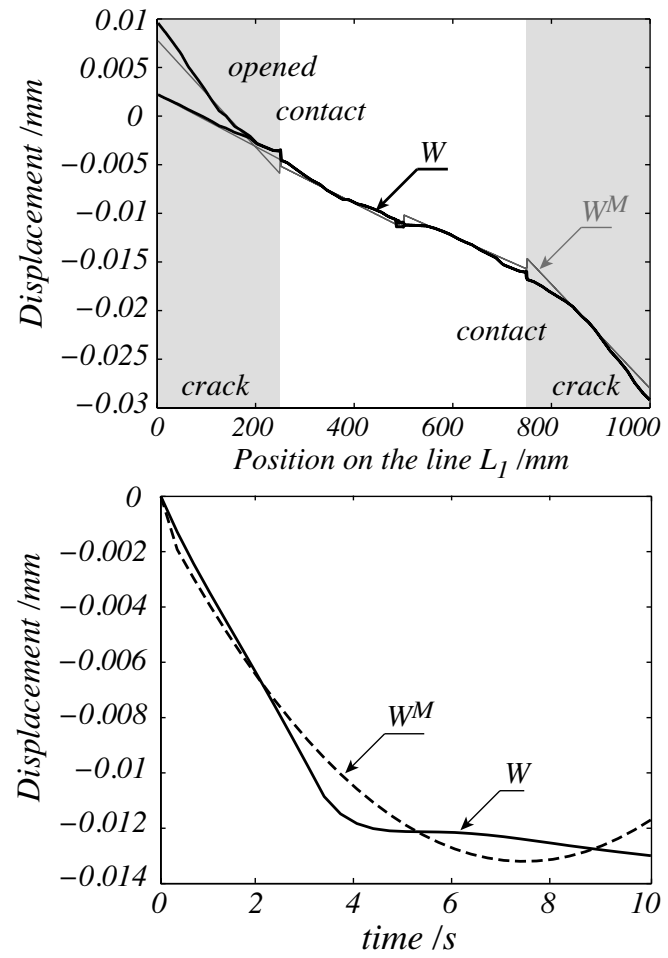

Figure 16: $\underline{W}$ and $\underline{W}^{M}$ over Line $L_{1}$ and over $[0, T]$

\section{Conclusions}

The multiscale computational strategy with space and time homogenization was presented. The efficiency and the scalability (which has not be discussed herein) of this approach make it well suited for parallel computing. The choice of macro basis has proved to be relevant. A new radial approximation technique for the resolution of the micro problems of the multiscale strategy has also been presented. This new technique involves the computation of approximately half the number of time functions needed by the previous approach and seems to be more robust. This technique not only reduces the number of computation of time functions, but increases its robustness. It also leads to the construction of a relevant basis of space functions, that can be reused along the iterations of the strategy. This new technique was implemented in a $3 \mathrm{D}$ viscoelastic code and mixed with the multiscale computational strategy.

\section{References}

[1] A. Ammar, B. Mokdad, F. Chinesta, and R. Keunings. A new family of solvers for some classes of multidimensional partial differential equations encountered in kinetic theory modeling of complex fluids. Journal of NonNewtonian Fluid Mechanics, 139(3):153-176, 2006.

[2] A. Ammar, B. Mokdad, F. Chinesta, and R. Keunings. A new family of solvers for some classes of multidimensional partial differential equations encountered in kinetic theory modeling of complex fluids: Part II: Transient simulation using space-time separated representations. Journal of Non-Newtonian Fluid $\mathrm{Me}$ chanics, 144(2-3):98-121, 2007.

[3] H. Ben Dhia and G. Rateau. The Arlequin method as a flexible engineering design tool. International Journal for Numerical Methods in Engineering, 62:1442-1462, 2005.

[4] R. A. Białecki, A. J. Kassab, and A. Fic. Proper orthogonal decomposition and modal analysis for acceleration of transient FEM thermal analysis. International Journal for Numerical Methods in Engineering, 62(6):774797, 2005.

[5] Anindya Chatterjee. An introduction to the proper orthogonal decomposition. Current Science, 78(7):808-817, 2000.

[6] F. Chinesta, A. Ammar, F. Lemarchand, P. Beauchene, and F. Boust. Alleviating mesh constraints: Model reduction, parallel time integration and high resolution homogenization. Computer Methods in Applied Mechanics and Engineering, 197:400-413, 2008.

[7] J.-Y. Cognard and P. Ladevèze. A large time increment approach for cyclic plasticity. International Journal of Plasticity, 9:114-157, 1993.

[8] J. Fish, K. Shek, M. Pandheeradi, and M. S. Shephard. Computational plasticity for composite structures based on mathematical homogenization: Theory and practice. Computer Methods in Applied Mechanics and Engineering, 148:53-73, 1997. 
[9] T. J. R. Hughes, G. R. Feijoo, L. Mazzei, and J.-B. Quincy. The variarional multiscale - a paradigm for computational mechanics. Computer Methods in Applied Mechanics and Engineering, 166:3-24, 1998.

[10] A. Ibrahimbegović and D. Markovič. Strong coupling methods in multi-phase and multiscale modeling of inelastic behavior of heterogeneous structures. Computer Methods in Applied Mechanics and Engineering, 192:30893108, 2003.

[11] P. Krysl, S. Lall, and J.E. Marsden. Dimentional model reduction in non-linear finite element dynamics of solids and structures. International Journal for Numerical Methods in Engineering, 51:479-504, 2001.

[12] P. Ladevèze. New algorithms: mechanical framework and development (in french). Technical Report 57, LMT-Cachan, 1985.

[13] P. Ladevèze. The large time increment method for the analyse of structures with nonlinear constitutive relation described by internal variables (in french). Comptes Rendus Académie des Sciences Paris, 309(II):1095-1099, 1989.

[14] P. Ladevèze. Nonlinear Computationnal Structural Mechanics - New Approaches and Non-Incremental Methods of Calculation. Springer Verlag, 1999.

[15] P. Ladevèze, L. Guitard, L. Champaney, and $\mathrm{X}$. Aubard. Debonding modeling for multidirectional composites. Computer Methods in Applied Mechanics and Engineering, 185:109$122,2000$.

[16] P. Ladevèze, D. Néron, and P. Gosselet. On a mixed and multiscale domain decomposition method. Computer Methods in Applied Mechanics and Engineering, 196:1526-1540, 2007.

[17] P. Ladevèze, D. Néron, and J.-C. Passieux. On multiscale computational mechanics with time-space homogenization. In Multiscale methods-Bridging the scales in Science and Engineering, chapter Space Time Scale Bridging methods. Oxford University Press. Ed. J. Fish, to appear 2009.

[18] P. Ladevèze and A. Nouy. On a multiscale computational strategy with time and space homogenization for structural mechanics. Computer Methods in Applied Mechanics and Engineering, 192:3061-3087, 2003.

[19] A. Nouy. A generalized spectral decomposition technique to solve a class of linear stochastic partial differential equations. Computer Methods in Applied Mechanics and Engineering, 196(45-48):4521-4537, 2007.

[20] A. Nouy and P. Ladevèze. On a multiscale computational strategy with time and space homogenization for structural mechanics. Computer Methods in Applied Mechanics and Engineering, 192:3061-3087, 2004.

[21] G. Picinbono, H. Delingette, and N. Ayache. Non-linear anisotropic elasticity for realtime surgery simulation. Graphical models, 56(5):305-321, 2003.

[22] D. Ryckelynck. A priori hyperreduction method: an adaptive approach. Journal of Computational Physics, 202:346-366, 2005.

[23] E. Sanchez-Palencia. Non homogeneous media and vibration theory. Lecture Notes in Physics, 127, 1980. 